\title{
Design and Development ofa Mirror Effect Control Prosthetic Hand with Force Sensing
}

\author{
Maryam Hanan Yahya, Norhazimi Hamzah*, Azizul Othman, Anis Diyana Rosli, \\ Rohaiza Baharudin, Adi Izhar Che Ani \\ Faculty of Electrical Engineering, UiTM Cawangan Pulau Pinang, Permatang Pauh, Malaysia \\ ${ }^{*}$ Cooresponding author, e-mail: norhazimi880@ppinang.uitm.edu.my
}

\begin{abstract}
Some of the already available prosthetic hands in the market are operated in open loop, without any feedback and expensive. This system counters those by having the prosthetic hand printed using $3 D$ printer and consist of a feedback sensor to make it a closed loop system. The system generally consists of two sections, mainly Finger Input and Prosthetic Output. The two sections communicate wirelessly for data transferring. The main purpose of the system is to control the prosthetic hand wirelessly using the Mirror Glove by performing a mirror effect that will translate movement from the glove onto the prosthetic hand. The Mirror Glove monitors the movements/bending of each fingers using force sensitive sensor. The prosthetic hand also has a sensor known as force sensitive resistor. The sensors will feedback the pressure on the prosthetic hand during object grasping, allowing the prosthetic hand to grasp delicate object without damaging it. Overall, the system will imitate the flex and relaxing of fingers inside the Mirror Glove and wirelessly control distant prosthetic hand to imitate the human hand.
\end{abstract}

Keywords: prosthetic hand, wireless control, 3D printer, force feedback, mirror effect

Copyright $\odot 2017$ Universitas Ahmad Dahlan. All rights reserved.

\section{Introduction}

There is a growing concern to accommodate for the disabled people in this growing modern technology world. There are many inventions made to cater for their needs. One of them includes a prosthetic designed for amputee whom lost the usage of their limbs due to accidents, birth complications or even diseases. Prosthetic by definition is an artificial device fitted to replace a missing body part. A prosthetic is designed for functional or cosmetic reasons or both [1].

The commercial prosthetic are rather expensive. This is due to the materials used for the artificial limb and the frequent contact hour with the attending doctor 2]. This cost issue motivates the development of a low-cost, effective anthropomorphic and dexterous hand that can beused for inexpensive prosthesis and basic research projectscompared to the other costly systems [3].

A prosthetic hand, besides the functionality of imitating real hand are also used for the treatment of Mirror Visual Feedback (MVF). MVF is a symptom suffered at the early stage of missing limb where the patient experience burning, cramping, crushing or lancinating known as phantom limb symptoms [4]. The painful sensation is purely made up by the brain as a way to cope with the loss. Thus, doctors use the MVF method to trick the brain that there is no missing limb and patient report that the pain significantly reduced after the treatment [4].

The most important part of a prosthetic hand is the hardware design. The desired functionality of prosthetic hand is determined by the capability of prosthetic hand to perform different postures as shown in Figure 1 [5]. It can be determined by the Degree of Freedom (DOF) of the prosthetic hand. In actual, human hand possess approximately 20 major DOF which allow it to execute a wide variety of grasp postures [6].The dexterity of the prosthetic is exhibited by imitating the natural motion of the human fingers [3]. Although, there has been some argument that as the number of degree of freedom increases, the control complexity of the hand will dramatically multiplies, which would result in dexterity degradation in term of hand performance [7]. 
Some of the commercially available prosthetic hand such asi-Limb are operated open loop, without force or position sensor[8]. Open loop movement are commonly repetitive, sequential and consist of simple steps[9]. However, the appropriate function of the prosthetic hand requires feedback to ensure it can adapt to the parameter of different object better. The absence of feedback lead to frustrating situation where grasped are inadvertently crushed or dropped [10].The proper forces required not to drop and not to break are important [11]. With feedback, the proper forces needed can be determined.

A previous study for a paper titled 'An Empirical Framework for Controlling Artificial Hand Gripper System using Smart Glove' use a glove that gathers data from flex sensor and transfer it to Arduino to move a servo motor on prosthetic hand. The proposed system have a feedback sensor attached onto the glove only [12].There is a disadvantages to this setup whereby if there is an object in the prosthetic hand palm but not in the glove, the servo motor (without feedback) on the prosthetic hand will unknowingly crush the object.

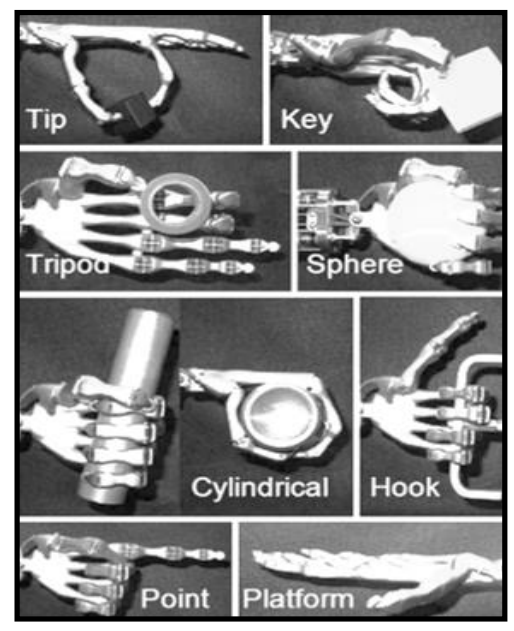

Figure 1. Grasp object

Hence, this project proposes the wireless control module to control prosthetic hand from another working hand using mirror effect with force feedback. It will counter the issue of uncontrolled force grasping by placing the pressure sensor on the prosthetic finger and measure actual pressure on object. The Finger Input and prosthetic hand will be communicating wirelessly using a radio frequency module to eliminate the messy condition wire and ensure smooth operation.

\section{Research Method}

The system is generally divided into two parts, where they are mutually connected by a wireless radio frequency module. The first part of the system would be the input circuit known as Finger Input. Glove was chosen to collect input data because it can provide a more accurate humanlike motions compared to other input such as joystick and dials [13]. The glove that collect the input data will be called as Mirror Glove.The second part is the output circuitcalled Prosthetic Output. The general system flow shown in Figure 2.

A few flex sensors are attached onto the Mirror Gloveto monitor the flexing and relaxing of each fingers. The data will then be transferred wirelessly to the prosthetic hand. The data will be processed on the second platform and servo motor will act as the muscle to move each fingers on prosthetic hand, mimicking the movements of fingers inside the glove. The feedback sensor placed on the prosthetic finger will monitor the pressure to ensure that the grip on the object is sufficient.

The system is separated into Finger Input and Prosthetic Output.Xbee will connect both circuit troughs the transmitting and receiving Bluetooth module.Both of the Finger Input and Prosthetic Output operate differently than the other. 


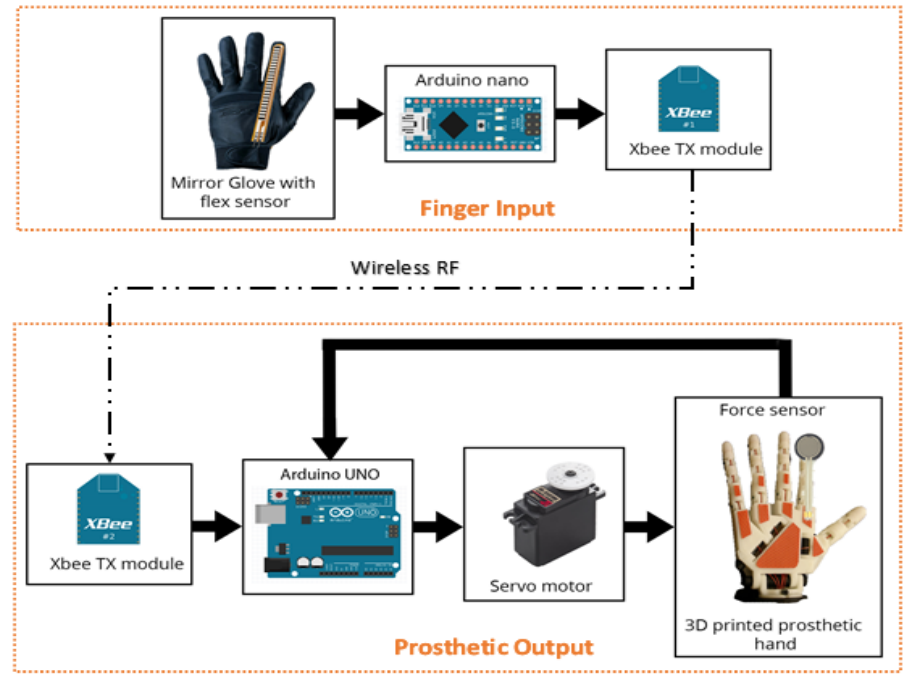

Figure 2. Overall system flow

\subsection{Finger Input}

Block diagram in Figure 3 shows the flow specified on the Finger Input only. The sensor (flex sensor) will be attached to the glove so that when the fingers bend inwards, the sensor will be able to calibrate the degree of flexing. It will then input the value to the Arduinonano and the microcontroller will transferred the data obtained to the other platform via Xbee wireless radio frequency (RF) module.

Based on the flow chart for Finger Input in Figure 4, initially the system will be in a completely OFF state until the switch button is ON. Then, the system will mainly monitor for any input from the sensor. In this situation, it would refer to the value of resistance output from the sensor and into the microcontroller as input. If there is any flexing detected, the data (resistance value) will be wirelessly transmitted to the second section of the system, which is the receiving module on the prosthetic hand. The input hardware was realized by sewing the flex sensors onto the Mirror Glove as and connected to distant processing unit strapped to the wrist as seen in Figure 5.

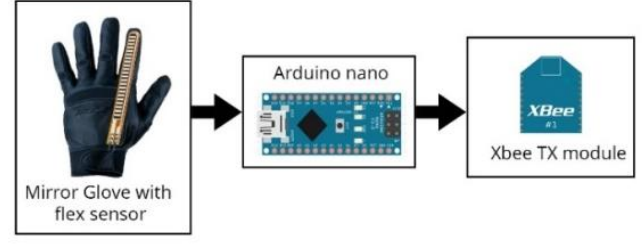

Figure 3. Finger Input block diagram

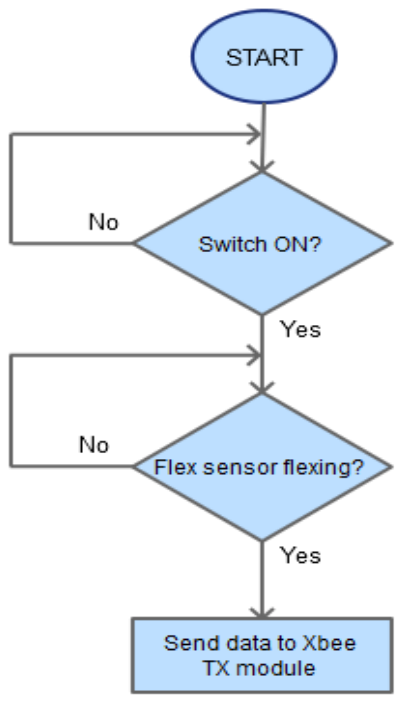

Figure 4. Finger Input flow chart 


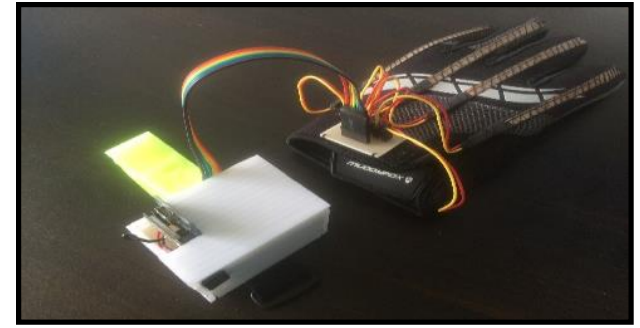

Figure 5. Mirror Glove

\subsection{Prosthetic Output}

The data received from the Xbee module on the Prosthetic Output part are processed and the necessary input signal into the motor are produced to move the fingers according to the glove movements. There is a Force sensor attached onto the prosthetic finger as shown in Figure 6 . The force sensor will be the feedback to the controller, to determine if there is any object detected in the palm as the fingers flexed inwards. If there is an object within the prosthetic hand's palm, the sensor will detect and notify the controller. From there onward, thecontroller will adjust the rotation of motor (finger) to properly grasp the object without causing any damage by putting excessive torque that could crush soft objects.

Flow chart of Prosthetic Output in Figure 7 shows that when the switch is ON, the microcontroller will immediately monitor for any incoming data from the transmitting module. The data received will be processed hence turning the motor clockwise or anticlockwise while trying to mirror the movements as accurately as possible. There will be a feedback sensor attached to the prosthetic fingers to detect whether if there are any objects to grasp and if so, the feedback will ensure that the pressure applied to the object is not excessive that could damage the object. In actual operation as in flowchart, when there is an object detected (pressure), the motor flexing the fingers will slow down (to ensure the object is grasp) and finally come to a stop at a comfortable object grip.

The prosthetic handhardware was printed using 3D printing technology and the material chosen was a multipurpose plastic known as ABS. ABS is known for its durability when compared to PLA. The hardware design was obtained from an open-source project called InMoov by Gael Langevin. Project InMoov is a complete robot that can be fully assembled form printed plastic and the .STL files are available online. The components printed are from the fingers until the forearm for the purpose of this study.

The hardware was assembled using all the material and components gathered. The final product is shown in Figure 8. The hardware represents a prosthetic from fingers to forearm of a prosthetic hand. Although the hardware only functions on the movement of fingers, there is a possibility to actuate the wrist for future improvement. There is a fishing line threaded through each fingers and connected to the motor located in the forearm. The fishing line here will act as the muscle that actuates the fingers.

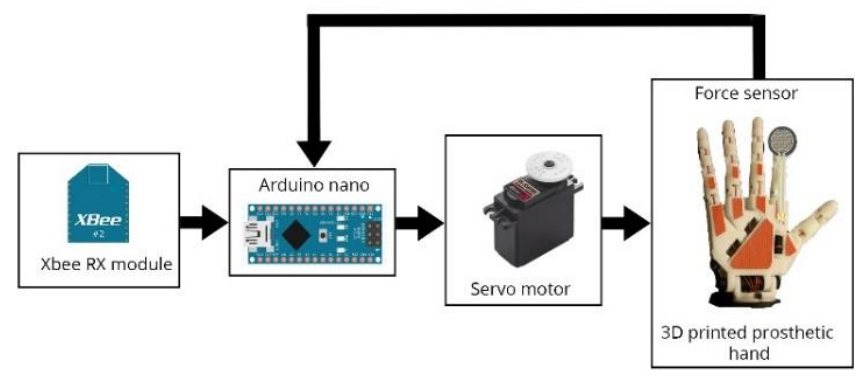

Figure 6. Prosthetic Output block diagram

TELKOMNIKA Vol. 15, No. 2, June 2017 : 949 - 956 


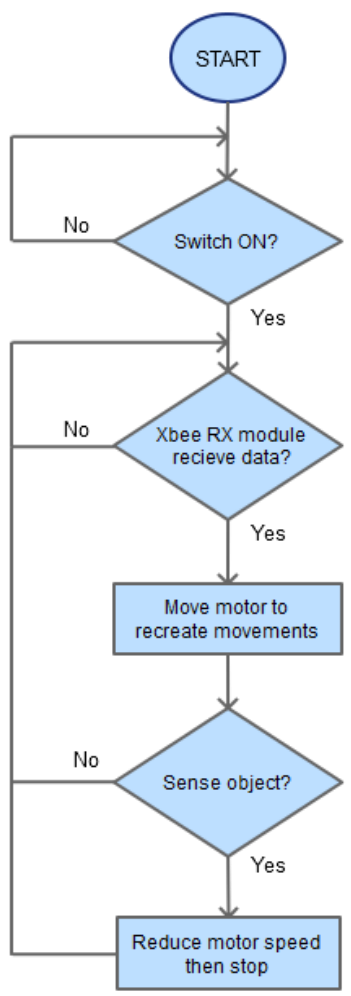

Figure 7. Output flowchart

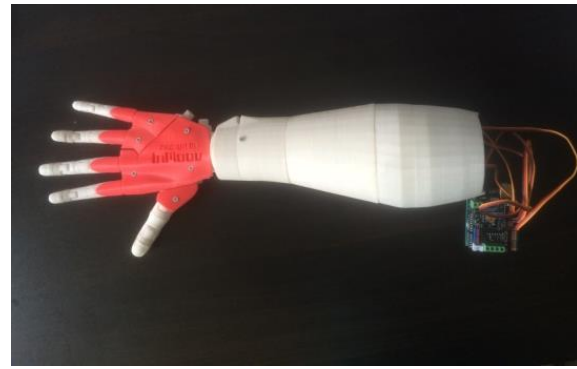

Figure 8. Prosthetic hand

\section{Results and Analysis}

Figure 9 show mirror movement between the Mirror Glove and prosthetic hand. The sensors on glove measure the degree of finger bend in the glove and transmit it to the prosthetic hand. The fingers on prosthetic hand are actuated using servo motor to create the mirror effect. Figure 10(a) show the relax condition while Figure 10(b) shows the condition during flexing. Manual measurement were done during relax and flexing conditionto obtain the degree of maximum bending for the fingers on the prosthetic hand. This was carried out to measure the accuracy and to determine the maximum bending capability of each fingers.

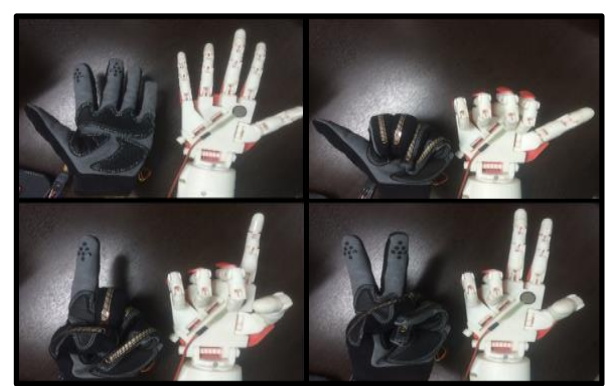

Figure 9. Mirror movement

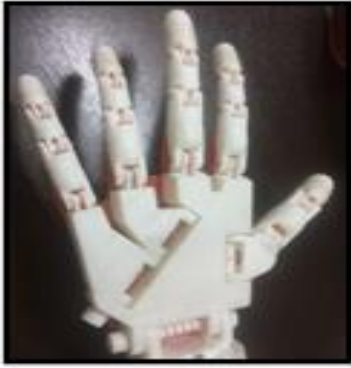

(a)

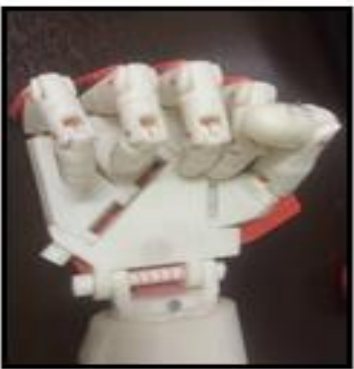

(b)

Figure 10. Relax and flex condition

Figure 11 shows angles $A, B$ and $C$ that were measured to record the maximum degree of bending for all the fingers on the prosthetic hand. The readings were taken using a specialized tool called Goniometeras in Figure 12. Measurement was taken with consideration that relax state is equal to zero degree. The measurement for maximum angle $A, B$ and $C$ during flexing are recorded in Table 1 . The angles during relax state were also measured and recorded in Table 2. 


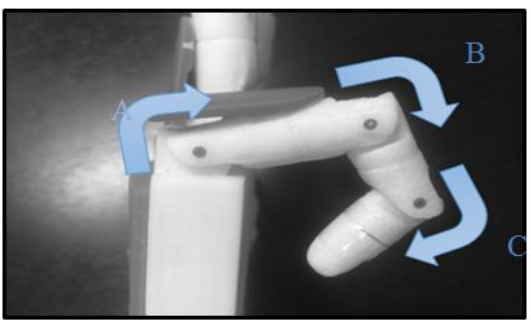

Figure 11. Measured angles

Table 1. Maximum flex angle

\begin{tabular}{llll}
\hline & A & B & C \\
\hline Thumb & $63^{\circ}$ & - & $75^{\circ}$ \\
Index & $101^{\circ}$ & $75^{\circ}$ & $73^{\circ}$ \\
Middle & $99^{\circ}$ & $76^{\circ}$ & $72^{\circ}$ \\
Ring & $87^{\circ}$ & $80^{\circ}$ & $76^{\circ}$ \\
Pinky & $71^{\circ}$ & $74^{\circ}$ & $70^{\circ}$ \\
\hline
\end{tabular}

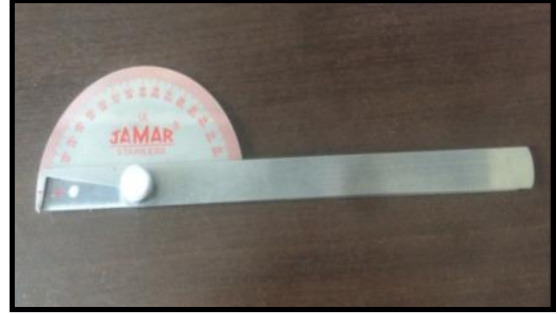

Figure 12. Goniometer

Table 2. Maximum relax angle

\begin{tabular}{llll}
\hline & A & B & C \\
\hline Thumb & $-5^{\circ}$ & - & $-9^{\circ}$ \\
Index & $1^{\circ}$ & $-6^{\circ}$ & $-14^{\circ}$ \\
Middle & $-0.5^{\circ}$ & $-2^{\circ}$ & $-7^{\circ}$ \\
Ring & $-5^{\circ}$ & $-4^{\circ}$ & $-18^{\circ}$ \\
Pinky & $-5^{\circ}$ & $-6^{\circ}$ & $-17^{\circ}$ \\
\hline
\end{tabular}

Since the hardware is made of printed ABS plastic, there are a few rigid parts with limited DOF. Often small printed plastic is not $100 \%$ accurate compared to the source file. One of the small part printed was the fingers. Thus, they do not fit perfectly with each other when assembled, resulting in a backward bending during relax state. Although the hardware were designed to have zero backward or forward bending during relax state. The prosthetic hand capability of grasping object grasp was tested. Figure 13 shows various shapes of object grasped by the prosthetic hand. Starting from the top left and clockwise, the shapes includes sphere, tip, cylinder and rectangular. All of these objects were tightly held and will not easily slip out of the hand.

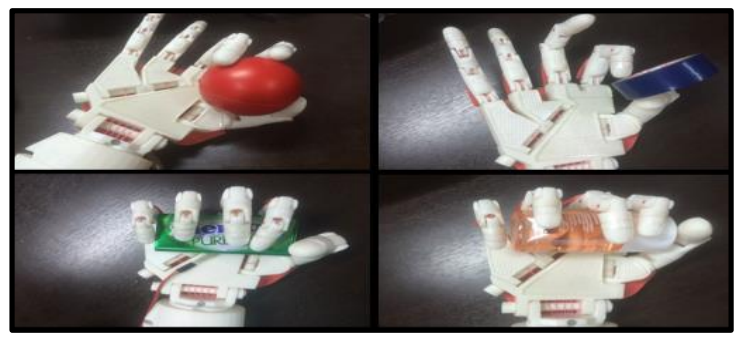

Figure 13. Grasp object

The final analysis was done on the effect of having a feedback sensor on the prosthetic hand. The test was done using a Play-Doh. Figure 14 (a) shows a Play-Doh placed onto the palm of the prosthetic hand and full flexing force was given. The effect was recorded and shown in Figure 15(a). Then, the same test was repeated with a feedback sensor as in Figure 14(b). The result in Figure 15(b) shows a significant difference of dent on the Play-Doh surface.

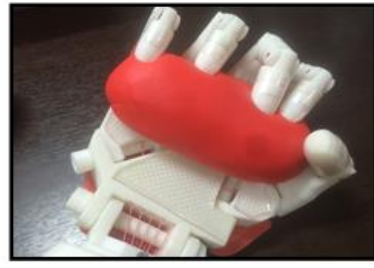

(a)

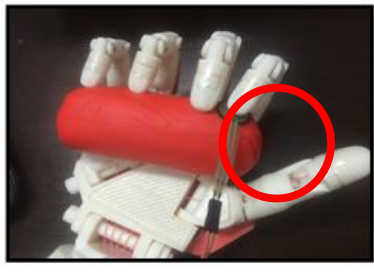

(b)

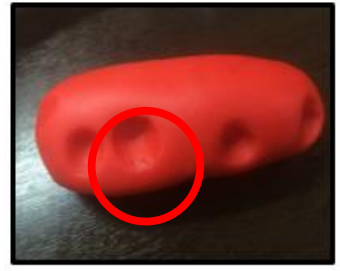

(a)

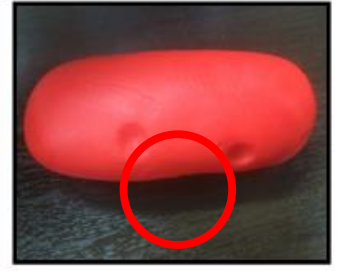

(b)

Figure 14. Grasp Play-Doh with and without feedback

Figure 15. Play-Doh dented with and without feedback 
The feedback threshold was determined by observing the prosthetic hand's firm grip and recording it as a threshold. If the pressure on the feedback sensor exceeds the threshold limit, the motor will stop exerting anymore force on the object to avoid crushing it. At this point, the controller will ignore any increasing flexing at Finger Input. The motor will only move if the input is to reduce the grip on the object. The pressure was monitored through a serial interface. Figure 16 and Figure 17 shows the graph representation of analogue value from pressure sensor without feedback and with feedback respectively.

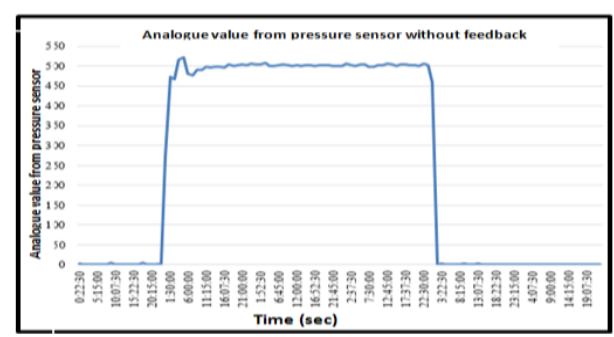

Figure 16. Analogue value from pressure sensor without feedback vs time

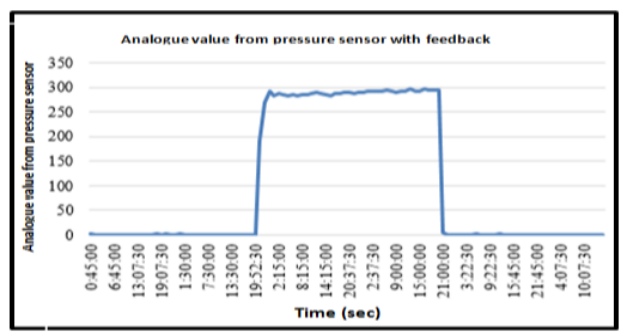

Figure 17. Analogue value from pressure sensor with feedback vs time

Based on the analogue value from pressure sensor logged in Figure 16, without any feedback sensor the motor will continuously exert force on the object where the highest value recoded at 521. By applying a feedback sensor, the analogue value from pressure sensor on grasped object can be capped below 300. The sensor will sense if the analogue value from pressure sensor exceeds 200, then it will allow a small delay to ensure object is securely grasped before forcing the motor (and finger) to stop. Thus, by having a feedback feature on prosthetic hand, actual pressure can be monitored to prevent delicate object crushed while still providing a firm grip.

\section{Conclusion}

The design and development of a wireless control prosthetic hand system use 3D printed prosthetic hand which reduced the cost while also being very reliable and customizable. It will wirelessly imitate the flex and relaxing of fingers inside Mirror Glove and wirelessly control distant prosthetic hand to imitate the human hand.The system is a closed loop whereby it incorporates a feedback. The feedback located on the prosthetic hand will calibrate the force exerted on the object and stop the motor so that the prosthetic hand can properly grasp delicate object without damaging it.

\section{Acknowledgements}

The authors would like to acknowledge Faculty of Electrical Engineering, UniversitiTeknologi MARA Pulau Pinang, and also Research Management Institute (RMI), UiTM and Ministry of Education for funding this research work under Research Acculturation Grant Scheme (RAGS) under the code 600-RMI/RAGS 5/3 (193/2014).

\section{References}

[1] Jokstad A, Orstavik J, Ramstad T. A definition of prosthetic dentistry. International Journal of Prosthodontics. 1998;11(4): 295-301.

[2] Briggs TW. Improving the quality of orthopaedic care within The National Health Service in England. Getting it Right First Time. Royal National Orthopaedic Hospital. 2012.

[3] Junaid A, Afzal MR, Rasheed T, Tahir S, Ahmed S, Sohail M, Ali M. Design and Control of Artificial Robotic Hand. International Conference on Robotics and Emerging Allied Technologies in Engineering (iCREATE). Islamabad. 2014: 10-15.

[4] Ramachandran VS, Altschuler EL. The use of visual feedback, in particular mirror visual feedback, in restoring brain function. Brain. 2009: 1693-1710. 
[5] Dalley SA, Wiste TE, Withrow TJ, Goldfarb M. Design of a Multifunctional Anthropomorphic Prosthetic Hand with Extrinsic Actuation. IEEE/ASME transactions on mechatronics. 2009:699-706.

[6] Dalley SA, Varol HA, Goldfarb M. A Method for the Control of Multigrasp Myoelectric Prosthetic Hands. IEEE Transactions on Neural Systems and Rehabilitation Engineering. 2012: 58-67.

[7] Liu H, Yang D, Jiang L, Fan S. Development of a Multi-DOF Prosthetic Hand with Intrinsic Actuation, Intuitive Control and Sensory Feedback. Industrial Robot: An International Journal. 2014; 41(4):38192.

[8] Engeberg ED. A Physiological Basis for Control of a Prosthetic Hand. Biomedical Signal Processing and Control. 2013; 8(1):6-15.

[9] Ekvall S, Kragic D. Interactive Grasp Learning Based on Human Demonstration. In Robotics and Automation, 2004. Proceedings. ICRA'04. 2004 IEEE International Conference on. 2004; 26(4): 3519-3524.

[10] Andrecioli R, Engeberg ED. Adaptive Sliding Manifold Slope Via Grasped Object Stiffness Detection with a Prosthetic Hand. Mechatronics. 2013; 23(8): 1171-1179.

[11] Jo J, Kim S, Oh Y, Oh SR. Contact Force Control of a Robotic Hand Using F / T Sensory Feedback with a Rigid Object. IEEE International Conference on Automation Science and Engineering (CASE). Seoul. 2012: 436-441.

[12] Ali AMM, Tomari R, Jamil MMA. An Empirical Framework for Controlling Artificial Hand Gripper System using Smart Glove. Procedia Computer Science. 2014; 42: 38-45.

[13] Fang B, Guo D, Sun F, Liu H, Wu Y. A Robotic Hand-Arm Teleoperation System using Human Arm/Hand with a Novel Data Glove. IEEE Conference on Robotics and Biomimetics. Zhuhai. 2015: 2483-2488. 